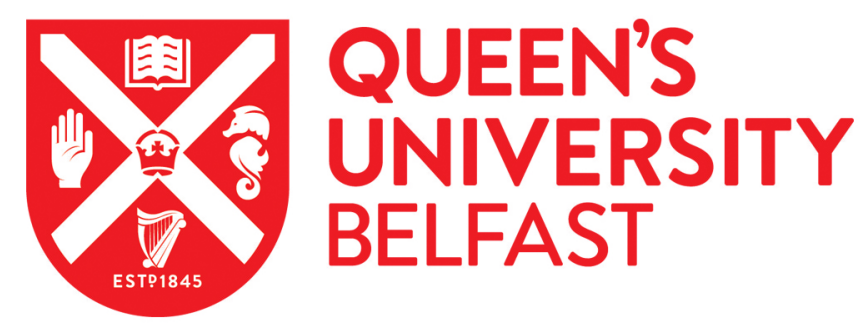

\title{
Relative Biological Effectiveness Variation Along Monoenergetic and Modulated Bragg Peaks of a 62-MeV Therapeutic Proton Beam: A Preclinical Assessment
}

Chaudhary, P., I Marshall, T., Perozziello, F. M., Manti, L., Currell, F. J., Hanton, F., McMahon, S. J., Kavanagh, J. N., Cirrone, G. A. P., Romano, F., Prise, K. M., \& Schettino, G. (2014). Relative Biological Effectiveness Variation Along Monoenergetic and Modulated Bragg Peaks of a 62-MeV Therapeutic Proton Beam: A Preclinical Assessment. International Journal of Radiation: Oncology - Biology - Physics, 90(1), 27-35. https://doi.org/10.1016/j.ijrobp.2014.05.010

Published in:

International Journal of Radiation: Oncology - Biology - Physics

\section{Document Version:}

Peer reviewed version

\section{Queen's University Belfast - Research Portal:}

Link to publication record in Queen's University Belfast Research Portal

\author{
Publisher rights \\ (C) 2014 Elsevier Inc. This manuscript version is made available under the CC-BY-NC-ND 4.0 licensehttp://creativecommons.org/licenses/by- \\ nc-nd/4.0/,which permits distribution and reproduction for non-commercial purposes, provided the author and source are cited
}

\section{General rights}

Copyright for the publications made accessible via the Queen's University Belfast Research Portal is retained by the author(s) and / or other copyright owners and it is a condition of accessing these publications that users recognise and abide by the legal requirements associated with these rights.

\section{Take down policy}

The Research Portal is Queen's institutional repository that provides access to Queen's research output. Every effort has been made to ensure that content in the Research Portal does not infringe any person's rights, or applicable UK laws. If you discover content in the Research Portal that you believe breaches copyright or violates any law, please contact openaccess@qub.ac.uk. 


\title{
RBE variation along monoenergetic and modulated Bragg peaks of a 62 $\mathrm{MeV}$ therapeutic proton beam: a pre-clinical assessment
}

Pankaj Chaudhary Ph.D. ${ }^{1^{*}}$, Thomas Marshall M.Sci. ${ }^{1^{*}}$, Francesca M. Perozziello M.Sc. ${ }^{3}$, Lorenzo Manti Ph.D. ${ }^{3}$, Frederick J. Currell Ph.D. ${ }^{2}$, F. Hanton M.Sci. ${ }^{2}$, Stephen J. McMahon Ph.D. ${ }^{1}$, Joy N. Kavanagh Ph.D. ${ }^{1}$, G.A.P. Cirrone Ph.D. ${ }^{4}$, Francesco Romano Ph.D. ${ }^{4}$, Kevin M. Prise Ph.D. ${ }^{1}$, Giuseppe Schettino Ph.D. ${ }^{1,5}$

* Equal Contribution

1.Centre for Cancer Research and Cell Biology, School of Medicine, Dentistry and Biomedical Sciences, Queen’s University Belfast, 97 Lisburn Road, BT97BL, Belfast, United Kingdom

2. Centre for Plasma Physics, School of Mathematics and Physics, Queen's University Belfast Belfast, BT7 1NN, UK

3. Department of Physics, University of Naples Federico II and INFN Naples Section University of Naples Monte S. Angelo, 80126 Naples, Italy

4. Istituto Nazionale di Fisica Nucleare, LNS, Via S. Sofia 62, Catania, Italy.

5. National Physical Laboratory, Hampton Road, Teddington, TW11 0LW, United Kingdom

Running Title: RBE variations along Proton beams

Acknowledgements: This research was financially supported by the Medical Research Council UK (Grant No. G 1100014), awarded to Drs. Giuseppe Schettino, Fred J Currell and Prof. Kevin M Prise. Travel costs to INFN-LNS were partly supported by EPSRC grant EP/H017844/1 and EU 262010 (ENSAR), awarded to GS and FJC.TM is thankful to the Department of Employment and Learning, Northern Ireland for providing fellowship. We are very thankful to our collaborators and the Users Support Group at INFN-LNS Catania, Italy.

Conflict of Interest: None

\author{
Corresponding Author \\ Prof. Kevin M Prise \\ Professor of Radiation Biology \\ Centre for Cancer Research \& Cell Biology \\ 97 Lisburn Road BT97BL Belfast, Northern Ireland, UK \\ Tel +44 (0) 2890972943 \\ Fax +44 (0) 2890972776 \\ Email: k.prise@qub.ac.uk
}




\section{Summary}

Biological optimization of proton therapy critically depends upon detailed evaluation of RBE variations along Bragg curve. Clinically accepted RBE value of 1.1 is an oversimplification, which disregards the steep rise of LET at the distal end of the SOBP. We observed significant cell killing RBE variations dependent upon beam modulation, intrinsic radiosensitivity and LET in agreement with the LEM predicted values indicating dose averaged LET as suitable

parameter for biological effectiveness. Data have also been used to validate a RBE parameterized model. 


\section{Abstract}

Purpose: The Biological optimization of proton therapy can only be achieved through a detailed evaluation of RBE variations along the full range of the Bragg curve. The clinically used RBE value of 1.1 represents a broad average, which disregards the steep rise of Linear Energy Transfer (LET) at the distal end of the Spread-Out Bragg Peak (SOBP). With particular attention to key endpoint of cell survival, our work presents a comparative investigation of cell killing RBE variations along monoenergetic (pristine) and modulated (SOBP) beams using human normal and radioresistant cells with the aim to investigate the RBE dependence on LET and intrinsic radiosensitvity.

Methods and Materials: Human fibroblasts (AG01522) and glioma (U87) cells were irradiated at six depth positions along pristine and modulated $62 \mathrm{MeV}$ proton beams at the INFN-LNS (Catania, Italy). Cell killing RBE variations were measured using standard clonogenic assays and were further validated using Monte Carlo simulations and the Local Effect Model (LEM).

Results: We observed significant cell killing RBE variations along the protons beam path, particularly in the distal region showing strong dose dependence. Experimental RBE values were in excellent agreement with the LEM predicted values indicating dose averaged LET as a suitable predictor of proton biological effectiveness. Data were also used to validate a parameterized RBE model.

Conclusions: The predicted biological dose delivered to a tumor region based on the variable RBE inferred from the data, varies significantly with respect to the clinically used constant RBE of 1.1. The significant RBE increase at the distal end suggests also a potential to enhance optimization of treatment modalities such as LET painting of hypoxic tumors. The 
study highlights the limitation of adoption of a constant RBE for proton therapy and suggests approaches for fast implementation of RBE models in treatment planning.

\section{Introduction}

Proton therapy is currently the fastest growing cancer treatment strategy attracting considerable interest from industry, the academic and the health care sector (1). Potential clinical advantages of proton beams are linked to the pattern of energy deposition termed the 'Bragg curve” which exhibits a well-defined, highly localized peak at the end of the proton track (2). Based upon the needs of clinical application, the Bragg peak can be spread out by modulating the proton energy in order to attain the desired uniform dose at depth throughout the target volume. The modulation in energy can be obtained by degrading or varying the entrance beam energy leading to superposition of several monoenergetic proton beams or pristine peaks of closely spaced energies known as the Spread Out Bragg Peak or SOBP (3, 4). Using protons or ion beams, it is therefore possible to obtain more defined dose distributions than those produced with photon beams, sparing a larger volume of healthy tissues from unwanted radiation exposure.

In addition to the favorable dose distributions made possible by the Bragg peak, successful implementation of any kind of ions used for radiotherapy critically depends on the relative biological effectiveness (RBE) (5). Whilst for energetic photons, the quality of induced damage does not change with depth and the total absorbed dose can be used as the main parameter to estimate the amount of damage produced, for charged particles the quality of the DNA lesions tend to become more clustered and complex along the particle track as the particle slows down (6). This is related to the clustering of ionizations that increases as the energy of the charged particles decreases. Estimation of the relative biological effectiveness 
(RBE) of proton beams compared to energetic X-rays is therefore a key issue in radiotherapy as any uncertainty in RBE translates directly into uncertainty of the biologically effective dose (i.e. physical dose $\times \mathrm{RBE}$ ) delivered to the patient, strongly undermining the $3.5 \%$ requirement for dose uncertainty in clinical settings $(7,8)$.

Current clinical practice adopts a constant RBE value of 1.1 across the entire SOBP irrespective of its size, beam modulation, depth, cellular radio-sensitivity and the delivered dose $(9,10)$. Furthermore, use of a single RBE value for protons is complicated as the RBE also depends on the dose per fraction, number of fractions, tissue types, level of oxygenation, and the biological end-point (11). In vitro studies reported proton RBE values increasing along the SOBP reaching 1.4-1.6 (12 - 15).

As shown by Frese et al in a modeling study where a theoretical variable RBE value is used to calculate an RBE-weighted proton treatment plan, there are some significant differences between the biologically weighted dose and the absorbed dose distributions for both the tumor and normal tissues (16). It is calculated that there may be as much as $3 \mathrm{~mm}$ increase in estimated range when a variable RBE weighting is used during treatment planning (17). These RBE variations are more important during fractionated exposure of hypoxic tumors. Good understanding of the dependency of RBE on the LET parameter and therefore the beam modulation may lead to further optimization of LET painting as effective tumor treatment modality $(18,19)$.

Studies carried out in the past addressing the issues of RBE variation along the proton path are mainly dominated by results obtained using non-human mammalian V79 cells or immortal human cells such as HeLa cells (20), which differ in radioresponse from the primary cells. Very few experiments report comparative findings from normal human 
primary and tumor cells in order to address the role of intrinsic radiosensitivity. Moreover, the conclusions are limited by the fact that the response was evaluated only at a few positions mainly mid SOBP $(21,22)$ and with large uncertainties on the depth positioning and therefore the delivered dose and LET. A more systematic approach supported by more precise measurements and a comparative analysis of both monoenergetic and modulated Bragg peak between human normal primary and radioresistant cell lines is still needed. Such studies will provide critical information for clinical treatment optimization algorithms and fundamental data for modeling studies.

In this work, we studied in detail the RBE variations in cell killing in two cell lines with different radiosensitivity (normal human skin fibroblasts (AG01522) and radioresistant human glioma (U87)) at several precise positions along a $62 \mathrm{MeV}$ modulated (SOBP) and monoenergetic (pristine) Bragg curve covering all the crucial depths-1.69, 28.21, 29.28, 29.76, 30.24, and $30.72 \mathrm{~mm}$ along pristine Bragg curve and 1.52,19.22, 24.28, 30.14, 30.82 and $31.22 \mathrm{~mm}$ along SOBP. Such depths correspond to positions of clinical relevance (i.e. entrance, proximal, central and distal end of a SOBP configuration) or where the LET changes rapidly (distal dose fall off of the Bragg peak in the pristine configuration). We used $62 \mathrm{MeV}$ as a starting point for higher energy studies however energies close to $60 \mathrm{MeV}$ have been successfully used for treating ocular melanoma and other superficial tumors.

\section{Methods and Material}

\section{Cell culture}

AG01522 cells were maintained in $\alpha$-modified Minimum Essential Medium (MEM) (Sigma Aldrich) supplemented with 20\% Fetal Bovine Serum (FBS) and 1\% penicillin-streptomycin (Gibco, Life Technologies Carlsbad, CA, USA). U87 cells were cultured in Dulbecco's 
Modified Eagle’s Medium (DMEM)-high glucose medium (Gibco, Life Technologies, Carlsbad, CA, USA) with $10 \%$ FBS and $1 \%$ penicillin-streptomycin. All cells were incubated in $5 \% \mathrm{CO}_{2}$ with $95 \%$ humidity at $37^{\circ} \mathrm{C}$. A detailed description is included in supplementary information (www.redjournal.org).

\section{Proton irradiation and dosimetry}

The Super-Conducting Cyclotron at the CATANA ocular melanoma treatment facility (Istituto Nazionale di Fisica Nucleare (INFN), Catania, Italy) generated a $62 \mathrm{MeV}$ proton beam. Water equivalent depths were simulated using high-grade Poly (methyl methacrylate) (PMMA) beam degraders (Goodfellows Ltd, Huntingdon, England) to $10 \mu \mathrm{m}$ precision with relative dose profiles obtained with a Markus ${ }^{\mathrm{TM}}$ electron ionization chamber $(100 \mu \mathrm{m}$ resolution). Detailed description of beam line and dosimetry has been previously published by Cirrone et al (23). For RBE determination, AG01522 and U87 cells at the same passage number were irradiated using $225 \mathrm{kV}_{\mathrm{p}}$ X-rays (XRAD 225, Precision X-ray Inc, New Haven CT, USA) at a dose rate of $0.591 \mathrm{~Gy} / \mathrm{min}$ in our laboratory in Queen’s University Belfast under similar conditions to the proton irradiations.

\section{Clonogenic assay}

After irradiation, cells were immediately trypsinized, counted and seeded onto six-well plates in duplicate with sufficient density to obtain $\sim 50$ colonies per well. Plates were then incubated in $5 \% \mathrm{CO}_{2}$ with $95 \%$ humidity at $37^{\circ} \mathrm{C}$ for $10-12$ days to allow for macroscopic colony formation. Colonies were fixed and stained using $0.5 \%$ crystal violet dye in $95 \%$ methanol in water for 30 minutes at room temperature then gently rinsed in water and air dried. Crystal violet stained colonies were counted manually in each duplicate well for each 
data point using Zeiss Stemi 2000 C stereomicroscope (Carl Zeiss, Germany). Colonies consisting of at least 50 cells were scored as viable.

\section{Data analysis and Simulation}

Cell survival and dose response data were fitted using the linear quadratic equation:

$S F=e^{-\left(\alpha D+\beta D^{2}\right)}$

Where SF denotes the Surviving Fraction of cells at dose D with curve fitting parameters $\alpha$ and $\beta$. Non-linear regression analysis was performed on survival curves using GraphPad Prism version 5.0c. RBE values were calculated relative to $225 \mathrm{kVp}$ X-rays according to

$$
R B E_{S F}=\frac{D_{S F}^{X}}{D_{S F}^{P}}
$$

where RBESF is the RBE at a survival level of $\mathrm{SF}$, and $D_{S F}^{X}$ and $D_{S F}^{P}$ are the X-ray and proton doses required to give a survival of SF, respectively. These dose values were calculated from the linear quadratic fit to the observed data. To allow for direct fitting of these dose values, and thus reduce fitting uncertainty on these terms, $\alpha$ and $\beta$ were re-stated in terms of $\mathrm{D}_{\mathrm{SF}}$ and $\gamma=\beta / \alpha$, as:

$$
\alpha=\frac{-\ln (S F)}{\left(D_{S F}\left(1+\gamma D_{S F}\right)\right)}, \beta=\gamma \alpha=\gamma \frac{-\ln (S F)}{\left(D_{S F}\left(1+\gamma D_{S F}\right)\right)}
$$

allowing for D $\mathrm{SF}$ and $\boldsymbol{\gamma}$ to be obtained explicitly at each survival level.

LET profiles were calculated from simulations using the Geant4 Monte Carlo toolkit (24) with Local Effect Model (LEM) comparisons using the methods described by Krämer et al (25). Using the LEM, the biological effect of radiation is determined based upon the 
local energy deposition in the cell nucleus, independent of the type of radiation. This independence allows the prediction of particle radiation effects based on cellular response under conventional photon modalities.

\section{Results}

\section{Depth dose and LET profile}

Depth, dose and LET values for the irradiation positions P1 to P6 along the $62 \mathrm{MeV}$ pristine peak and SOBP are shown in supplementary table-1 (www.redjournal.org). Dose and LET profiles at various depths in water are reported in Figure 1. It is evident that although beam modulation causes an increase in relative dose at the entrance position P1 ( $60 \%$ of the peak dose vs. $\sim 20 \%$ in the monoenergetic scenario) the LET remains unchanged. For the monoenergetic configuration, LET reaches $11.9 \mathrm{keV} / \mu \mathrm{m}$ at the position of peak dose P4 and $22.6 \mathrm{keV} / \mu \mathrm{m}$ at the most distal position P6. Similarly, LET increases across the SOBP reaching a peak of $25.9 \mathrm{keV} / \mu \mathrm{m}$ at the most distal position P6.

\section{Cell survival response curves}

Figure 2 shows the survival curves of AG01522 and U87 cells irradiated along the monoenergetic (left) and modulated (right) proton beams. X-ray survival shows relatively more shoulder for U87 cells $\left(\alpha=0.11 \pm 0.028 \mathrm{~Gy}^{-1} ; \beta=0.06 \pm 0.01 \mathrm{~Gy}^{-2}\right)$ than for AG01522 cells $\left(\alpha=0.54 \pm 0.06 \mathrm{~Gy}^{-1} ; \beta=0.062 \pm 0.02 \mathrm{~Gy}^{-2}\right)$, requiring a larger dose range (0-7 Gy) to cover survival fractions between 0.01 and 1 . Survival curves become progressively steeper and more linear towards the distal region, particularly for the AG01522 cell line, correlating with an increasing LET and supporting the hypothesis of increased DNA damage complexity.

\section{RBE dependency on depth and dose}


Figure 3 shows the RBE comparison ( $\mathrm{SF}=50 \%, 10 \%$ and $1 \%$ ) at various depths for both cell lines and dose profiles. RBE increase is apparent in all cases at the distal end, reaching values well above the clinically used RBE of 1.1. Figure 3 also indicates the critical effect of survival level on RBE, with an almost two-fold increase in maximum RBE between the $1 \%$ and $50 \%$ surviving fractions. To further elaborate on the effect of proton dose on RBE Figure 4 plots RBE as a function of dose at each position for both cell lines. Consistent with the trend in Figure 3, RBE at all positions is elevated for lower doses (corresponding to a higher survival fraction). This is particularly evident for the normal cells and at the distal positions, which could have a substantial impact when considering safety margins in treatment planning and varying the dose per fraction.

\section{RBE variation as a function of LET}

Figure 5 reports RBE variation as a function of LET for both cell lines and beam configurations. A clear RBE increase is observed with LET in all scenarios and, within the experimental uncertainties and limited LET range of protons, the data are well described by a linear response. Although the monoenergetic RBE values appear consistently higher than for the SOBP, comparisons of the data with the LEM show that despite the different energy spectra as shown in the Supplementary Figure 1 (www.redjournal.org), the RBE in both configurations is adequately described by the same linear response with the $\mathrm{R}^{2}$ value (co-efficient of determination indicating goodness of fit of a function) ranging from 0.82 0.98. Using the clinically implemented rapid calculation method of the LEM, weighted $\alpha$ and $\beta$ values based on the energy spectrum at each experimental position are used to calculate RBE values. While assuming a nuclear radius $\approx 6.5 \mu \mathrm{m}$, in accordance with typical human skin fibroblast and glioma dimensions $(26,27)$, the threshold dose parameter 
(Dt) was optimized at 9.5 and 8 Gy by independently fitting to the experimentally obtained data for the AG01522 and U87 cell lines respectively.

\section{Parameterization of RBE}

To fully appreciate the impact of dose, LET and intrinsic radiosensitivity, the RBE can be parameterized. By adopting the linear quadratic formulation and the RBE definition (RBE $=\mathrm{D}_{\mathrm{X} \text {-ray }} / \mathrm{D}_{\text {Proton }} @$ isoeffect), the RBE can be expressed as a function of the $\alpha$ and $\beta$ parameters and the delivered dose

$$
\left.\operatorname{RBE}=\left(\left(\alpha_{\mathrm{x}}^{2}+4 \beta_{\mathrm{x}} \mathrm{D}_{\mathrm{p}}\left(\alpha_{\mathrm{p}}+\beta_{\mathrm{p}} \mathrm{D}_{\mathrm{p}}\right)\right)^{\wedge}(1 / 2)-\alpha_{\mathrm{x}}\right)\right) /\left(2 \beta_{\mathrm{x}} \mathrm{D}_{\mathrm{p}}\right)
$$

Where $\alpha_{\mathrm{x}}, \beta_{\mathrm{x}}, \alpha_{\mathrm{p}}$ and $\beta_{\mathrm{p}}$ are the $\alpha$ and $\beta$ parameter from the X-ray and proton exposure and $D_{p}$ is the proton delivered dose. The $\alpha$ and $\beta$ for each position are shown in the Table- 1 of the supplementary information available at (www.redjournal.org). Several equivalent expressions can be also derived. The LET dependency can be explicitly included by analyzing the variation of $\alpha_{\mathrm{p}}$ and $\beta_{\mathrm{p}}$ for the different depth and LET position investigated. From the supplementary Figure 2 (www.redjournal.org), it is reasonable to assume that the $\alpha$ parameter varies linearly with LET in case of both monoenergetic Bragg peak and SOBP. However, the slope for SOBP is lower than that of pristine beam suggesting that small variations in the ionization clustering (i.e. LET) can result in significant effects on $\alpha$ parameter. Furthermore, the $\beta$ parameter varied non-significantly with LET for both monoenergetic and SOBP leading to a constant $\beta$ assumption in our RBE parameterization. For the RBE parameterization we have used an average $\beta$ value estimated by averaging the $\beta$ values for all positions (average $\beta$ values for AG01522 $=0.051 \pm 0.038$; U87 $=0.059 \pm$ 0.024). This is in broad agreement with the previously reported data (28-30) obtained using proton beams of specific energies (which in principle is different than using a 
monochromatic beam and placing samples at different depths). The $\alpha$ parameter is therefore expressed as

$$
\alpha_{\mathrm{p}}=\alpha_{\mathrm{x}}+\lambda \mathrm{LET}
$$

whilst the $\beta$ parameter is assumed to remain constant with LET $\left(\beta_{\mathrm{p}}=\beta_{\mathrm{x}}\right)$. From the above equation, is also evident that small variations of the $\beta$ parameter have no effect.

The final RBE parameterization model can therefore be expressed as

$$
\left.\operatorname{RBE}=\left(\left(\alpha_{\mathrm{x}}^{2}+4 \beta_{\mathrm{x}} \mathrm{D}_{\mathrm{p}}\left(\alpha_{\mathrm{x}}+\lambda \mathrm{LET}+\beta_{\mathrm{x}} \mathrm{D}_{\mathrm{p}}\right)\right)^{\wedge}(1 / 2)-\alpha_{\mathrm{x}}\right)\right) /\left(2 \beta_{\mathrm{x}} \mathrm{D}_{\mathrm{p}}\right)
$$

with the $\lambda$ parameter for our cell system of $0.0451 \mu \mathrm{m} \mathrm{keV}^{-1} \mathrm{~Gy}^{-1}$ and $0.0127 \mu \mathrm{m} \mathrm{keV}-1$ $\mathrm{Gy}^{-1}$ for the AG01522 and U87 respectively.

To assess critical discrepancies between the experimental RBE and accepted clinical RBE, figure 6 compares RBE weighted dose calculated using the constant (RBE 1.1) and experimental variable RBE. With the application of variable RBE in the SOBP regime, AG0 and U87 cell lines respectively see an increase of $18.3 \%$ and $17.9 \%$ in RBE weighted dose in the SOBP region, extending the effective range by $130 \mu \mathrm{m}$ and $150 \mu \mathrm{m}$. The most marked increase occurs in the distal dose fall off region (DDF) with 79\% increase in the region beyond the biological dose with clinical RBE .

\section{Discussion}

Using a systematic approach with particular emphasis on dosimetry to assess critical variations, the dataset produced by this study provides a reference in the ongoing debate of fixed versus variable RBE in proton therapy. Moreover, generation of radiobiological monoenergetic datasets will aid modeling techniques as a basis of treatment planning (31). In agreement with radiobiological models, data reported (figure-2) indicate that cellular response in terms of cell death varies along monoenergetic proton beams as well as the 
SOBP of a modulated beam, reaching the highest values towards the distal end of the Bragg peak (12-15). Survival curves become progressively steeper and more linear towards the distal region, particularly for the AG01522 cell line, correlating with a rising LET and supporting the hypothesis of increased damage complexity. Differences are observed across the SOBP with the RBE consistently higher than the clinical used value of 1.1. Using Hep2 cells, Britten et al reported RBE differences between proximal, middle and distal end of the SOBP with a value of 2.3 at distal dose fall off (22). Small differences observed in the RBE-LET relationship between monoenergetic and modulated beams could be attributed to the different energy spectra experienced by the samples for the same dose averaged LET. The Local Effect Model simulations are in excellent agreement with the experimental data and confirm that small non-significant differences between the monoenergetic and modulated beam are to be expected. Similar small differences between monoenergetic and modulated beam response were reported by Belli et al in V79 cells (12) and attributed to energy spectra of secondary species. However, on follow up studies using carbon ions, Belli et al reported that the SOBP is less effective than monoenergetic beams of the same dose averaged LET. The differences were also dependent on the specific cell line leading to the conclusion that the dose averaged LET might not be a suitable parameter for predicting the biological effectiveness of heavy ion beams. Under the experimental conditions reported in this study, the dose averaged LET appears to be a suitable parameter to predict RBE for cell killing along a proton beam but most likely this cannot be extrapolated to heavier ions like carbon. In particular considering the limited LET range covered in a proton beam, a linear relationship between RBE and LET appears to be valid for each cell model and dose level. 
It is important to note that RBE values strongly depend on the survival level at which the calculations are performed (i.e. absorbed dose) and cellular radio-sensitivity with larger values obtained for the $50 \%$ level and dramatically decreasing at the $1 \%$ level (32). This is particularly critical for hyper-fractionation strategies and heterogeneous cell populations where higher survival levels are expected following an individual radiation exposure. Considering that clinical radiotherapy fractions deliver $\sim 2$ Gy to tumor cells which correspond to survival levels in the range $30-60 \%$ for the U87 cancer cells investigated, the adoption of a constant $\mathrm{RBE}=1.1$ appears to be limitative and inadequate. Adopting RBE values calculated at the 2 Gy level (or at the level of the delivered dose/fraction) might be a better clinical option. The RBE-Dose relationship reported in figure 4 further indicates how rapidly the RBE changes with dose especially in the low dose region which would characterize the tumor-healthy tissue boundaries. The RBE variation effect is more marked in the radio-sensitive cell line (AG01522).

The biologically effective dose profile calculated using AG01522 data obtained in this study, indicates an underestimation of the biologically effective dose delivered to the surrounding normal and tumor area of up to 24 and $37 \%$ respectively by the treatment employing RBE=1.1. Although extrapolation from in vitro cell line data to in vivo tissues is not straightforward, these results highlight the extent of potential underestimation resulting from the adoption of fixed RBE values. Furthermore, the distal edge of the SOBP might be shortened by $150 \mu \mathrm{m}$ for radioresistant-U87 cells and $130 \mu \mathrm{m}$ for normal AG01522 cells using the fixed RBE value. Such differences become even more critical for different fractionation modalities where the dose per fraction can be significantly lower than $2 \mathrm{~Gy}$. 
The RBE differences observed in this study support the hypothesis that employment of variable RBE might lead to significant optimization of proton therapy and closer outcome predictions as also shown by Dasu (18). Our study also highlights how current technology and dosimetry can provide the necessary support for RBE measurements with an accuracy level suitable for clinical purposes and further extrapolation to higher energies relevant for treating tumors with greater volumes. Finally, the use of a monochromatic beam as reference appears to be a valid approach offering a useful tool and allowing comparative investigations. 


\section{References}

1. Suit HD, Goitein M, Munzenrider J, et al. Increased efficacy of radiation therapy by use of proton beam. Strahlenther Onkol 1990; 166:40-4.

2. Karger CP and Jakel O. Current status and new developments in ion therapy. Strahlenther Onkol 2007; 183:295-300.

3. Fokas E, Kraft G, Engenhart-Cabillic R. Ion beam radiobiology and cancer: time to update ourselves. Biochim Biophys Acta 2009; 1796:216-229.

4. Weber U, Kraft G. Design and construction of a ripple filter for a smoothed depth dose distribution in conformal particle therapy. Phys Med Biol 1999; 44:2765-2775.

5. Levin WP, Kooy H, Loeffler J.S et al. Proton beam therapy. British J Cancer 2005; 93:849-854.

6. Goodhead D. T. Energy deposition stochastics and track structure: what about the target? Radiat Prot Dosim 2006; 122(1-4): 3-15.

7. Brahme, A. Development of radiation therapy optimization. Acta Oncol 2000; 39(5): 579-95.

8. Paganetti H, Niemierko A, Ancukiewicz M, et al. Relative biological effectiveness (RBE) values for proton beam therapy. Int J Radiat Oncol Biol Phys 2002; 53:407-21

9. Jones, B, Underwood, TSA, and Dale RG. The potential impact of relative biological effectiveness uncertainty on charged particle treatment prescriptions. The British J Radiol 2011: 84; 61-69.

10. Blomquist E, Russell KR, Stenerlow B et al. Relative biological effectiveness of intermediate energy protons. Comparison with ${ }^{60} \mathrm{Co}$ gamma-radiation using two cell lines. Radiother Oncol 1993; 28:44-51. 
11. Mohan R, Mahajan, A and Minsky BD. New strategies in radiation therapy: exploiting the full potential of protons. Clin Cancer Res 2013; 19(23); 6338-43

12. Belli M, Bettega D, Calzolari P, et al. Inactivation of human normal and tumour cells irradiated with low energy protons. Int J Radiat Biol 2000; 76:831- 839.

13. Robertson JB, Williams JR, Schmidt RA, et al. Radiobiological studies of a highenergy modulated proton beam utilizing cultured mammalian cells. Cancer 1975; 35:16641677.

14. Courdi A, Brassart N, Herault J, et al. The depth-dependent radiation response of human melanoma cells exposed to 65-MeV protons. Br J Radiol 1994; 67:800-4.

15. Wouters, BG, Lam G K, Y., Oelfke, U et al. Measurements of relative biological effectiveness of the $70 \mathrm{MeV}$ proton Beam at TRIUMF using Chinese Hamster V79 cells and the high-precision cell sorter assay. Radiat Res 1996; 146: 159-170

16. Frese MC, Wilkens JJ, Huber PE, et al. Application of constant vs. variable relative biological effectiveness in treatment planning of intensity- modulated proton therapy. Int $J$ Radiat Oncol Biol Phys 2011; 79:80-8.

17. Carabe A, Moteabbed M, Depauw N, et al. Range uncertainty in proton therapy due to variable biological effectiveness. Phys Med Biol 2012; 57:1159-1172.

18. Dasu, A. Impact of variable RBE on proton fractionation. Med Phys 2013; 40(1): $11705-11714$.

19. Bassler N, Jäke O, Søndergaard CS, et al. Dose and LET-painting with particle therapy. Acta Oncol 2010; 49(7): 1170-1176 
20. Friedrich T, Scholz U, ElsäSser T et al. Systematic analysis of RBE and related quantities using a database of cell survival experiments with ion beam irradiation. $J$ Radiat Res 2012; 1-21

21. Petrovic I, Ristic-Fira A, Todrovic D et al. Response of radio resistant human melanoma cell line along the proton spread out Bragg peak. Int J Radiat Biol 2010; 86(9): 742-751.

22. Britten RA, Nazaryan V, Davis KL, et al. Variations in the RBE for cell killing along the depth dose profile of a modulated proton therapy beam. Radiat Res 2013; 179: 21-28. 23. Cirrone G. A. P. et al. A 62-MeV Proton Beam for the Treatment of Ocular Melanoma at Laboratori Nazionali del Sud-INFN. IEEE Trans Nucl Sci 2004; 51, 3568-3662

24. Cirrone GAP, Cuttone G, Mazzaglia S, et al. Hadrontherapy: a Geant4-Based Tool for Proton/Ion-Therapy Studies. Prog in Nuc Sci and Technol 2011: 2: 207-212.

25. Krämer M and Scholz M. Rapid calculation of biological effects in ion radiotherapy. 2006; Phys Med Biol 51; 1959

26.Suzuki, M., Kase, Y., Yamaguchi, H., Kanai, T. \& Ando, K. Relative biological effectiveness for cell-killing effect on various human cell lines irradiated with heavy-ion medical accelerator in Chiba (HIMAC) carbon-ion beams. Int. J. Radiat. Oncol. 48, 241250 (2000).

27.Elsässer, T., Krämer, M. \& Scholz, M. Accuracy of the local effect model for the prediction of biologic effects of carbon ion beams in vitro and in vivo. Int. J. Radiat. Oncol. Biol. Phys. 71, 866-72 (2008).

28. Wilkens JJ, Oelfke U. Three-dimensional LET calculations for treatment planning of proton therapy. Med Phys 2004; 14:41-46. 
29. Hawkins RB. A microdosimetric-kinetic theory of the dependence of the RBE for cell death on LET. Med Phys 1998; 25; 1157.

30. Dale RG, Jones B. The assessment of RBE effects using the concept of biologically effective dose. Int J Radiat Oncol Biol Phys1999; 43(3): 639-45.

31. Jones B, Underwood T SA, Fernandez AC et al. Fast neutron relative biological effects and implications for charged particle therapy. British J Radiol 2011; 84; S11-S18.

32. Gerweck, LE and Kozin SV. Relative biological effectiveness of proton beams in clinical therapy. Radiother Oncol 1999; 50; 135-142. 


\section{Figure Legends:}

Figure 1. Dose and LET profiles for $62 \mathrm{MeV}$ monoenergetic (top) and modulated (bottom) proton beam configurations. Vertical lines mark cell irradiation positions P1 - P6. Relative dose and GEANT4 derived dose averaged LET values are indicated in dashed and solid black lines respectively.

Figure 2. Clonogenic survival data at experimental irradiation positions P1 - P6 for AG01522 and U87 cells in monoenergetic and modulated $62 \mathrm{MeV}$ proton beam configurations. Error bars indicate standard error of the mean.

Figure 3. Comparison of RBE as a function of depth along monoenergetic and modulated $62 \mathrm{MeV}$ proton beams for the normal fibroblast (AG01522, top panel) and radioresistant glioma (U87, bottom panel) cells. RBE values are relative to $225 \mathrm{kV}_{\mathrm{p}} \mathrm{X}$-rays using proton $\alpha$ and $\beta$ values obtained using the Linear-Quadratic model. Error bars indicate standard error of the mean.

Figure 4. RBE variation as a function of dose and experimental position for normal fibroblast (AG01552) and radioresistant glioma (U87) cells after exposure to a monoenergetic $62 \mathrm{MeV}$ proton beam. Lines are for visual guidance only. Error bars indicate standard error of the mean.

Figure 5. RBE variation as a function of LET, comparing monoenergetic and modulated $62 \mathrm{MeV}$ proton beam configurations in normal fibroblast (AG01522, top panel) and radioresistant glioma (U87, bottom panel) cells at survival levels of 50\%, 10\% and 1\%. Lines are for visual guidance only. Error bars indicate standard error of the mean.

Figure 6. Comparison of RBE weighted or biological dose (the product of physical dose and RBE, which is expressed as $\mathrm{Gy}(\mathrm{RBE})$, calculated using clinical constant and 
experimental variable RBE values. Application of variable RBE resulted in an increase of RBE weighted dose in the tumor region (i.e. SOBP plateau), approximately by $18.1 \%$ and $17 \%$ for the normal AG01522 and radioresistant U87 cells respectively. RBE escalations in the distal region result in 130 and $150 \mu \mathrm{m}$ extension of the effective SOBP distal edge for the AG01522 and U87 cell lines. 
Figure-1
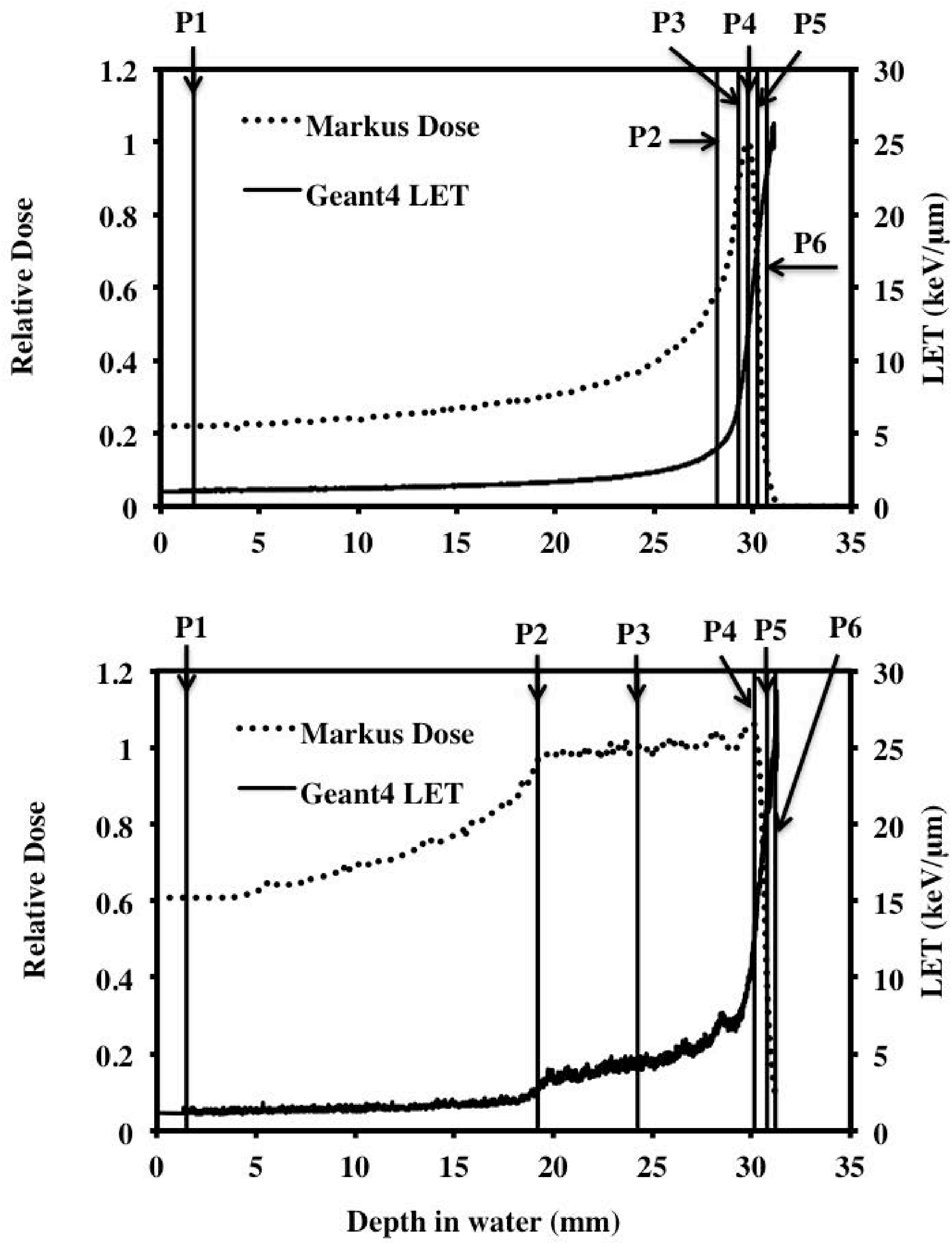

Figure 1. Dose and LET profiles for $62 \mathrm{MeV}$ monoenergetic (top) and modulated (bottom) proton beam configurations. Vertical lines mark cell irradiation positions P1 - P6. Relative dose and GEANT4 derived dose averaged LET values are indicated in dashed and solid black lines respectively. 
Figure-2
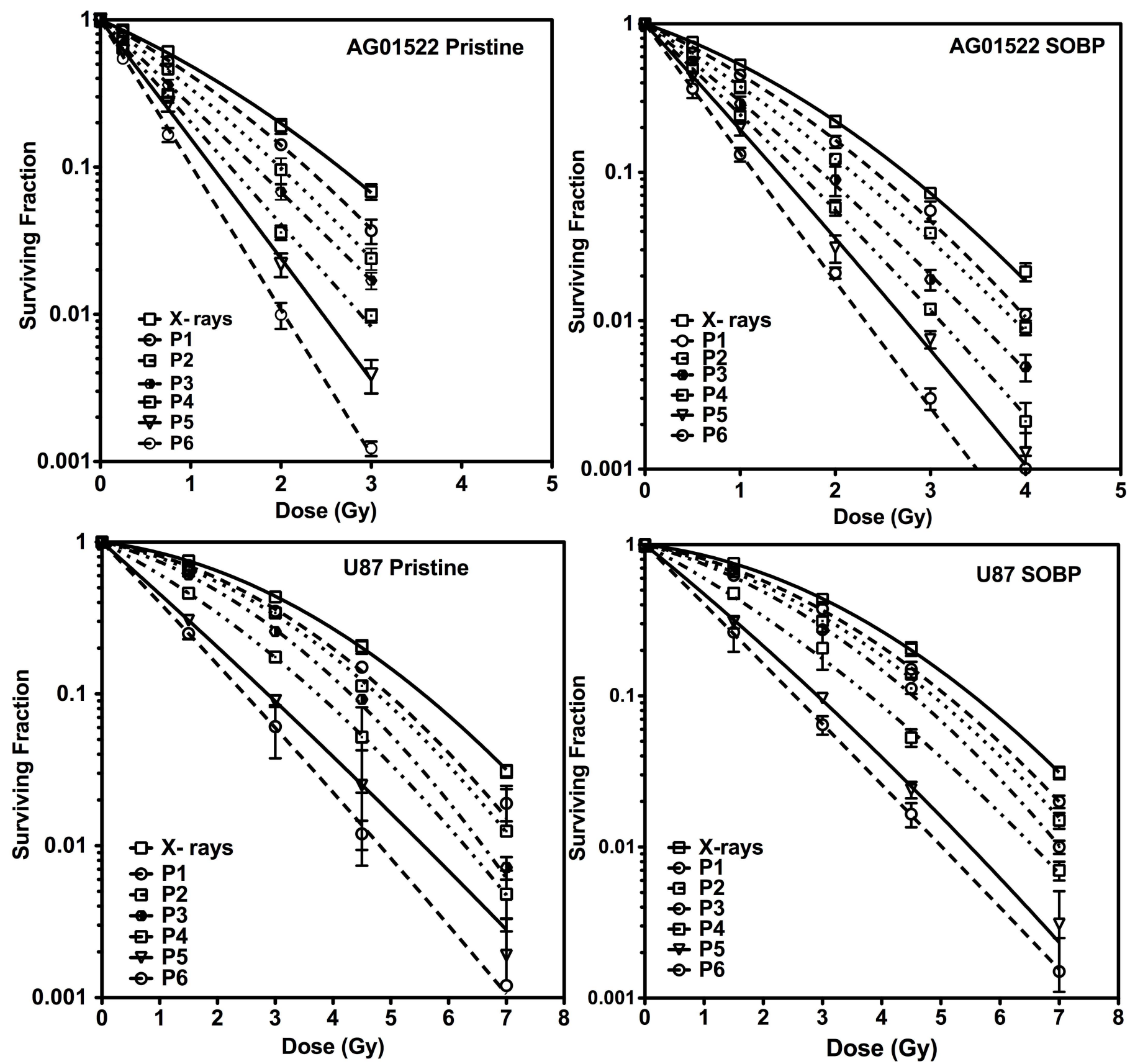

Figure 2. Clonogenic survival data at experimental irradiation positions P1 - P6 for AG01522 and U87 cells in monoenergetic (left panels) and modulated SOBPof $62 \mathrm{MeV}$ proton beam configurations (right panels). Error bars indicate standard error of the mean (SEM). 
Figure-3
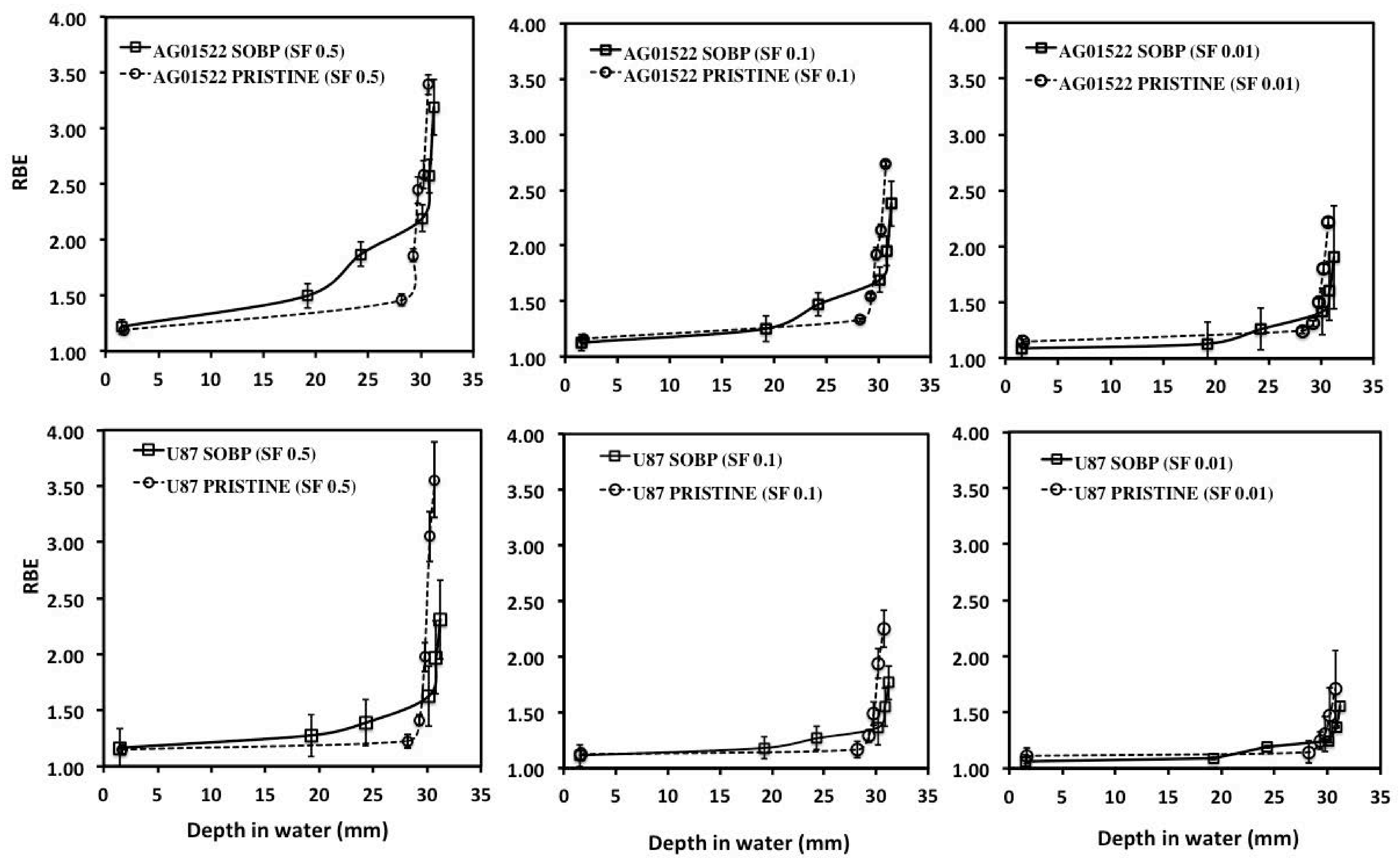

Figure-3.Comparison of RBE as a function of depth along monoenergetic and modulated $62 \mathrm{MeV}$ proton beams for the normal fibroblast (AG01522, top panel) and radioresistant glioma (U87, bottom panel) cells. RBE values are relative to $225 \mathrm{kVp} \mathrm{X}$-rays using proton $\alpha$ and $\beta$ values obtained using the Linear-Quadratic model. Error bars represent \pm standard error of the mean. 
Figure-4
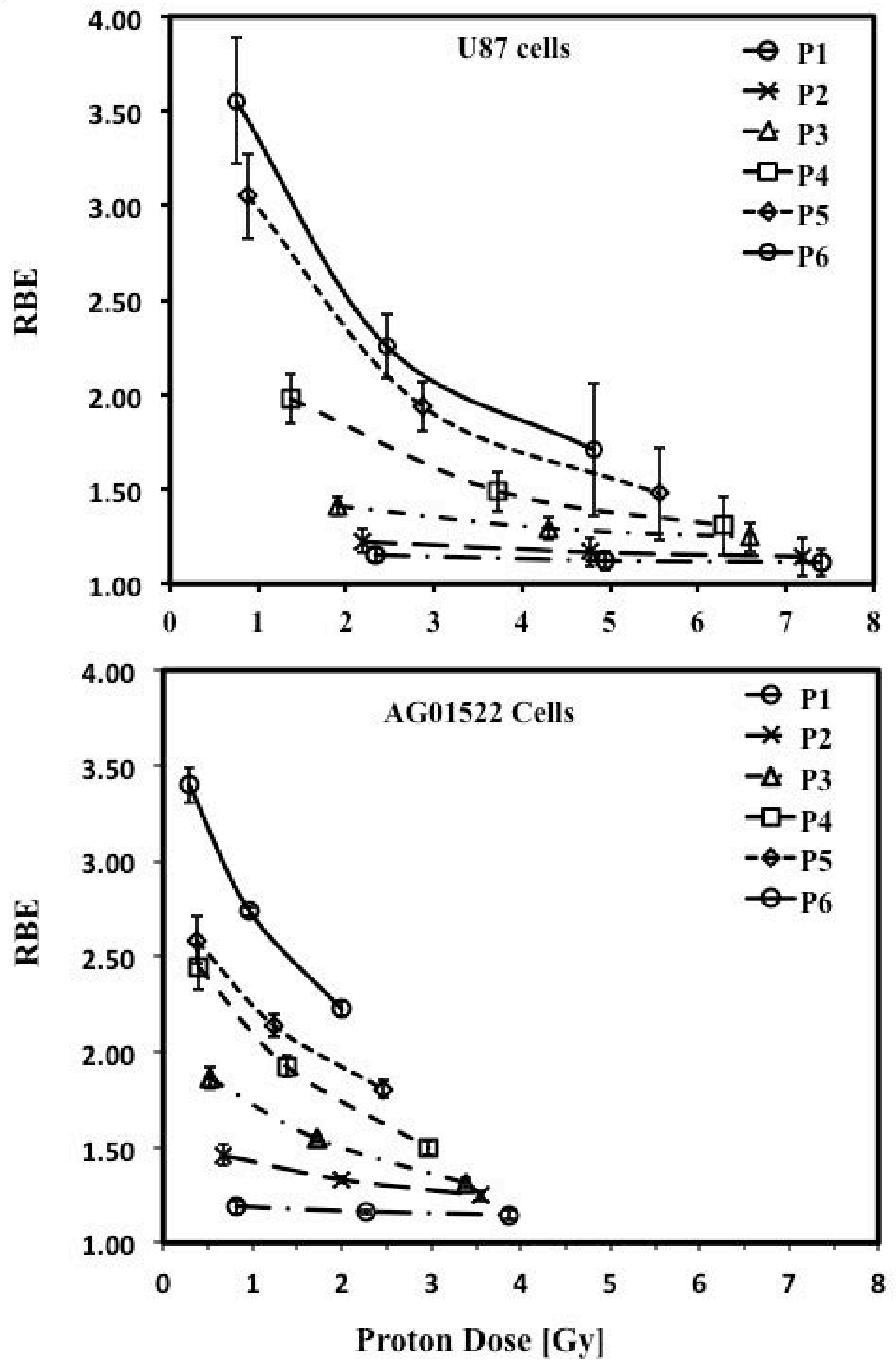

Figure 4. RBE variation as a function of dose and experimental position for normal fibroblast (AG01552) and radioresistant glioma (U87) cells after exposure to a monoenergetic $62 \mathrm{MeV}$ proton beam. Lines are for visual guidance only. Error bars represent standard error of the means. 


\section{Figure-5}
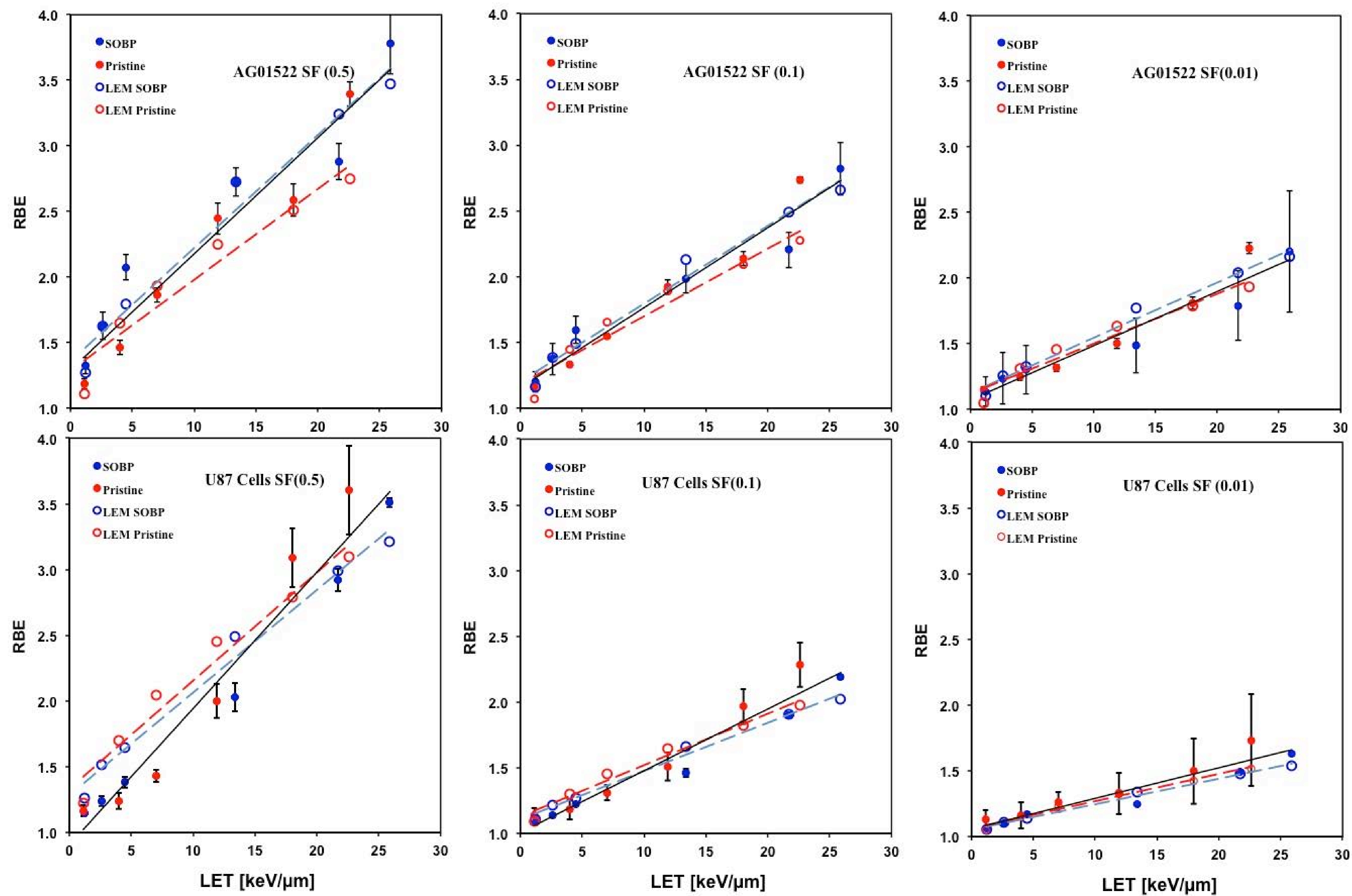

Figure 5. RBE variation as a function of LET, comparing monoenergetic and modulated $62 \mathrm{MeV}$ proton beam configurations in normal fibroblast (AG01522, top panel) and radioresistant glioma (U87, bottom panel) cells at surviving levels of $50 \%, 10 \%$ and $1 \%$. Lines are for visual guidance only. Error bars indicate standard error of the mean. 


\section{Figure-6}

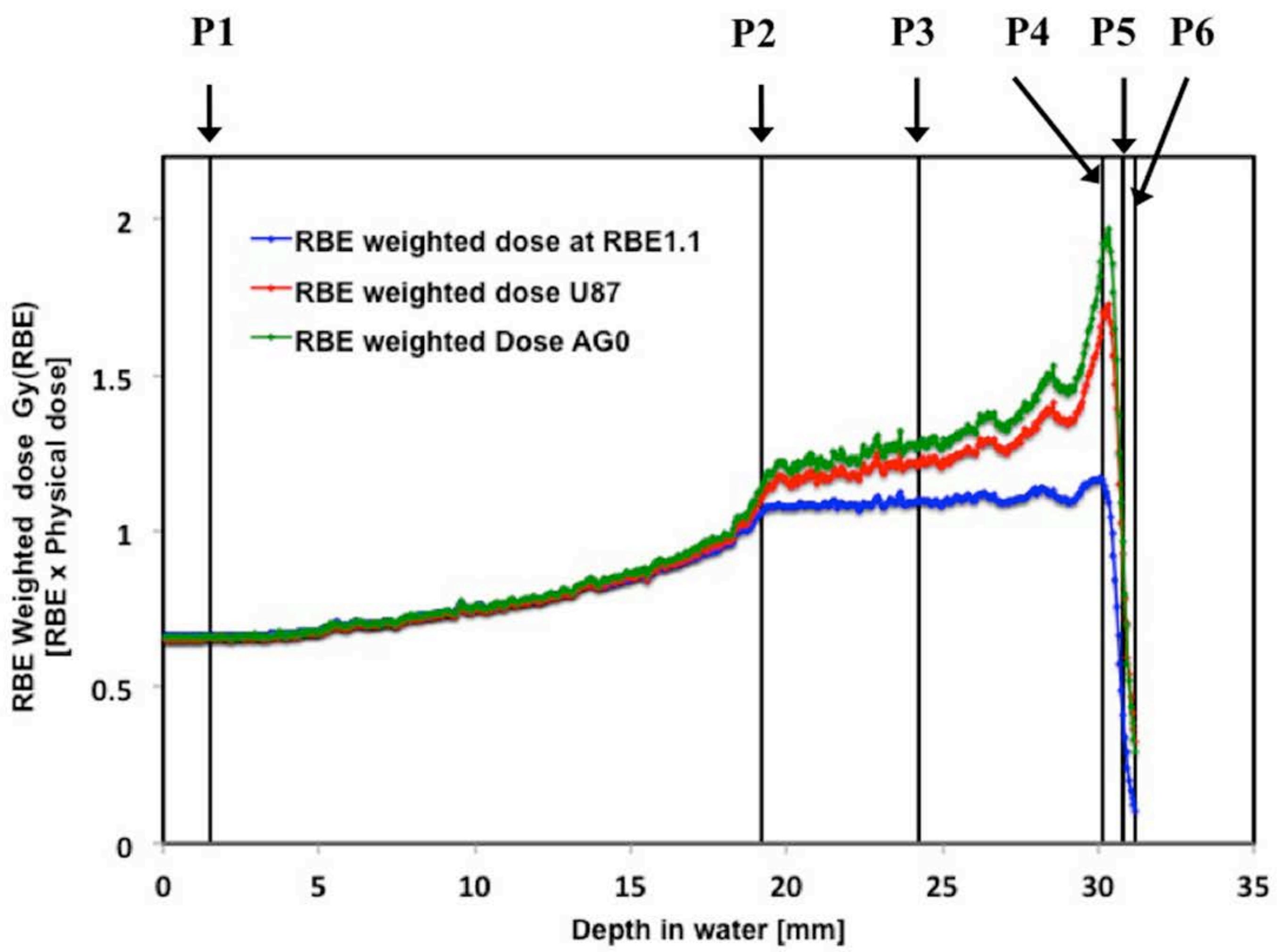

Figure 6. Comparison of RBE weighted or biological dose (the product of physical dose and $R B E$ which is expressed as $\mathrm{Gy}(\mathrm{RBE})$, calculated using clinical generic RBE1.1 (blue line) and experimental variable (green for AG0 and red for U87 cells) RBE values. Application of variable RBE resulted in an increase of biological dose in the tumor region (i.e. SOBP plateau), increasing by approximately $17 \%$ and $18 \%$ for the normal AG01522 and radioresistant U87 cells respectively. RBE escalations in the distal region result in 130 and $150 \mu \mathrm{m}$ extension of the effective SOBP distal edge for the AG01522 and U87 cell lines. 


\section{Supplementary Information}

\section{Materials and Methods}

Cells handling and transportation. For proton beam line experiments $80-90 \%$ confluent flasks were completely filled with warm $\left(37^{\circ} \mathrm{C}\right)$ low serum $(2.5 \%)$ medium, then sealed and packed in polystyrene foam containers. Upon arrival at the facility, low serum medium was replaced with regular full growth medium specific to the respective cell lines, as described. The flasks were incubated for 24 -36 hours to allow recovery from any stress occurring during transportation. For each sample polystyrene slide flasks of 1mm thickness and growth area $9 \mathrm{~cm}^{2}$ were seeded with $3 \times 10^{5}$ cells in $3 \mathrm{ml}$ complete medium (Thermo Scientific Nunc, catalogue no. 170920) and incubated in $5 \% \mathrm{CO}_{2}$ at $37^{\circ} \mathrm{C} 24$ hours before irradiation. Immediately before irradiation, full serum cell culture medium was replaced with low serum medium before being transported in a polystyrene foam box to the target area. Samples were mounted in sets of 5 on a remotely controlled X-Y axis translator. All samples were exposed at room temperature..

\section{Proton Irradiation and Dosimetry}

Reference dosimetry was carried out using a parallel plate Markus ionization chamber (Advanced Markus Chamber, $0.02 \mathrm{~cm}^{2}$, type 34045, PTW, Freiberg, Germany), calibrated according to the standards stated by the IAEA Technical Report Series 2000 code of practice. Samples were irradiated at water equivalent depths simulated using high-grade Poly(methyl methacrylate) (PMMA) beam degraders (Goodfellows Ltd., Huntingdon England) to $10 \mu \mathrm{m}$ precision. The SOBP (11 mm plateau) was generated by the same modulator wheel used in routine clinical procedures. Cells were irradiated at an average dose rate of $3 \mathrm{~Gy} / \mathrm{min}$ with a $1.7 \times 1.7 \mathrm{~cm}^{2}$ collimated proton beam of $>80 \%$ uniformity. Dosimetry and dose uniformity was further validated using Gafchromic (EBT2) film densitometry.

\section{X-ray Irradiation and Dosimetry}

For RBE determination AG01522 and U87 cells at the same passage number were irradiated using $225 \mathrm{kV}_{\mathrm{p}}$ X-rays (XRAD 225, Precision X-ray Inc, New Haven CT, USA) 
at a dose rate of $0.591 \mathrm{~Gy} / \mathrm{min}$ in our laboratory in Queen's University Belfast under similar conditions to the proton irradiations.

\section{Simulations}

The LET profile in a voxelized water phantom, representing the experimental slide flask assembly, was inferred from simulations using the Geant4 Monte Carlo toolkit [28]. The simulation geometry consisted of an accurate reconstruction of the INFN proton transport beamline, including all elements using the "hadrontherapy" advanced example. Dose averaged LET was attained by the calculation of the local mean of the proton stopping

power, weighted by the local energy spectrum. $10^{5}$ primary events were required to give acceptable statistics for each simulation.

\section{Local Effect Model Calculations}

Details of the Local Effect Model (LEM) have been reported in a number of publications. Using the LEM, the biological effect of radiation is determined based upon the local energy deposition in the cell nucleus, independent of the type of radiation. This independence allows the prediction of particle radiation effects based on cellular response under conventional photon modalities. Using the clinically implemented rapid calculation method of the LEM, weighted $\alpha$ and $\beta$ values based on the energy spectrum at each experimental position are used to calculate RBE values. While assuming a nuclear radius $\approx 6.5 \mu \mathrm{m}$, in accordance with typical human skin fibroblast and glioma dimensions, the threshold dose parameter $\mathrm{D}_{\mathrm{t}}$ was optimized at 9.5 and $8 \mathrm{~Gy}$ by independently fitting to the experimentally obtained data for the AG0 and U87 cell lines respectively. 
Table-1 Depth, LET and survival parameters characteristics for U87 and AG01522 cells

Pristine

\begin{tabular}{|l|c|c|l|c|c|c|}
\hline Positions & $\begin{array}{c}\text { Depth } \\
(\mathbf{m m})\end{array}$ & $\begin{array}{c}\mathbf{L E T} \\
(\mathbf{k e V} / \mathbf{\mu m})\end{array}$ & $\boldsymbol{\alpha}\left(\mathbf{G y}^{-\mathbf{1}}\right) \pm$ Error & $\boldsymbol{\beta}\left(\mathbf{G y}^{-2}\right) \pm$ Error & $\boldsymbol{\alpha}\left(\mathbf{G y}^{-\mathbf{1}}\right) \pm$ Error & $\boldsymbol{\beta}\left(\mathbf{G y}^{-2}\right) \pm$ Error \\
\hline P1 & 1.69 & $1.11 \pm 0.03$ & $0.14 \pm 0.02$ & $0.064 \pm 0.009$ & $0.75 \pm 0.10$ & $0.119 \pm 0.081$ \\
P2 & 28.21 & $4.02 \pm 0.16$ & $0.17 \pm 0.04$ & $0.065 \pm 0.017$ & $1.02 \pm 0.13$ & $0.061 \pm 0.013$ \\
P3 & 29.28 & $7.0 \pm 0.9$ & $0.22 \pm 0.02$ & $0.071 \pm 0.009$ & $1.29 \pm 0.15$ & $0.041 \pm 0.012$ \\
P4 & 29.76 & $11.9 \pm 1.5$ & $0.44 \pm 0.06$ & $0.045 \pm 0.002$ & $1.70 \pm 0.12$ & $0.079 \pm 0.013$ \\
P5 & 30.24 & $18.0 \pm 1.4$ & $0.77 \pm 0.08$ & $0.008 \pm 0.003$ & $1.87 \pm 0.20$ & $0.074 \pm 0.025$ \\
P6 & 30.72 & $22.6 \pm 1.2$ & $0.90 \pm 0.13$ & $0.010 \pm 0.006$ & $2.43 \pm 0.28$ & $0.057 \pm 0.048$ \\
& & & & & & \\
\hline
\end{tabular}

U87

AG01522
SOBPU87

\begin{tabular}{|l|c|c|c|c|c|c|}
\hline Positions & $\begin{array}{c}\text { Depth } \\
(\mathbf{m m})\end{array}$ & $\begin{array}{c}\text { LET } \\
(\mathbf{k e V} / \boldsymbol{\mu m})\end{array}$ & $\boldsymbol{\alpha}\left(\mathbf{G y}^{-\mathbf{1}}\right) \pm$ Error & $\boldsymbol{\beta}\left(\mathbf{G y}^{-2}\right) \pm$ Error & $\boldsymbol{\alpha}\left(\mathbf{G y}^{-\mathbf{1}}\right) \pm$ Error & $\boldsymbol{\beta}\left(\mathbf{G y}^{-2}\right) \pm$ Error \\
\hline P1 & 1.52 & $1.2 \pm 0.2$ & $0.16 \pm 0.04$ & $0.056 \pm 0.015$ & $0.66 \pm 0.07$ & $0.117 \pm 0.005$ \\
P2 & 19.22 & $2.6 \pm 0.2$ & $0.19 \pm 0.04$ & $0.058 \pm 0.051$ & $0.89 \pm 0.11$ & $0.075 \pm 0.008$ \\
P3 & 24.28 & $4.5 \pm 0.3$ & $0.22 \pm 0.04$ & $0.064 \pm 0.016$ & $1.15 \pm 0.09$ & $0.047 \pm 0.007$ \\
P4 & 30.14 & $13.4 \pm 1.5$ & $0.31 \pm 0.08$ & $0.056 \pm 0.035$ & $1.36 \pm 0.09$ & $0.037 \pm 0.009$ \\
P5 & 30.82 & $21.7 \pm 1.5$ & $0.41 \pm 0.09$ & $0.056 \pm 0.042$ & $1.61 \pm 0.12$ & $0.023 \pm 0.013$ \\
P6 & 31.22 & $25.9 \pm 2.6$ & $0.5 \pm 0.07$ & $0.064 \pm 0.033$ & $2.01 \pm 0.22$ & $0.011 \pm 0.002$ \\
& & & & & & \\
\hline
\end{tabular}

\section{AG01522}


Supplementary Figure 1. Energy spectra at the different experimental positions for the monoenergetic (top panel) and modulated (bottom panel) $62 \mathrm{MeV}$ proton beam.

\section{Pristine Peak Energy Spectrum}

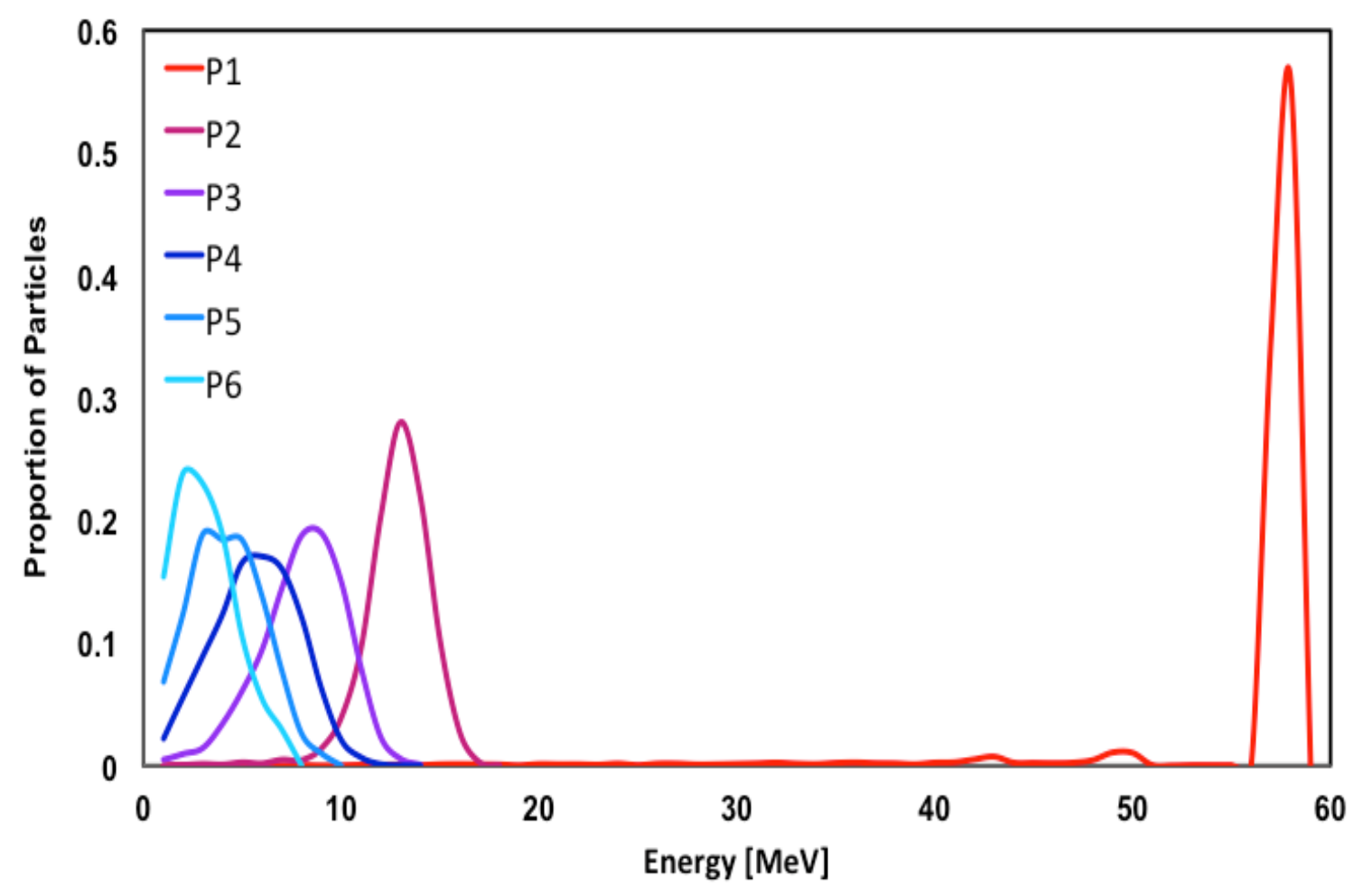

SOBP Energy Spectrum

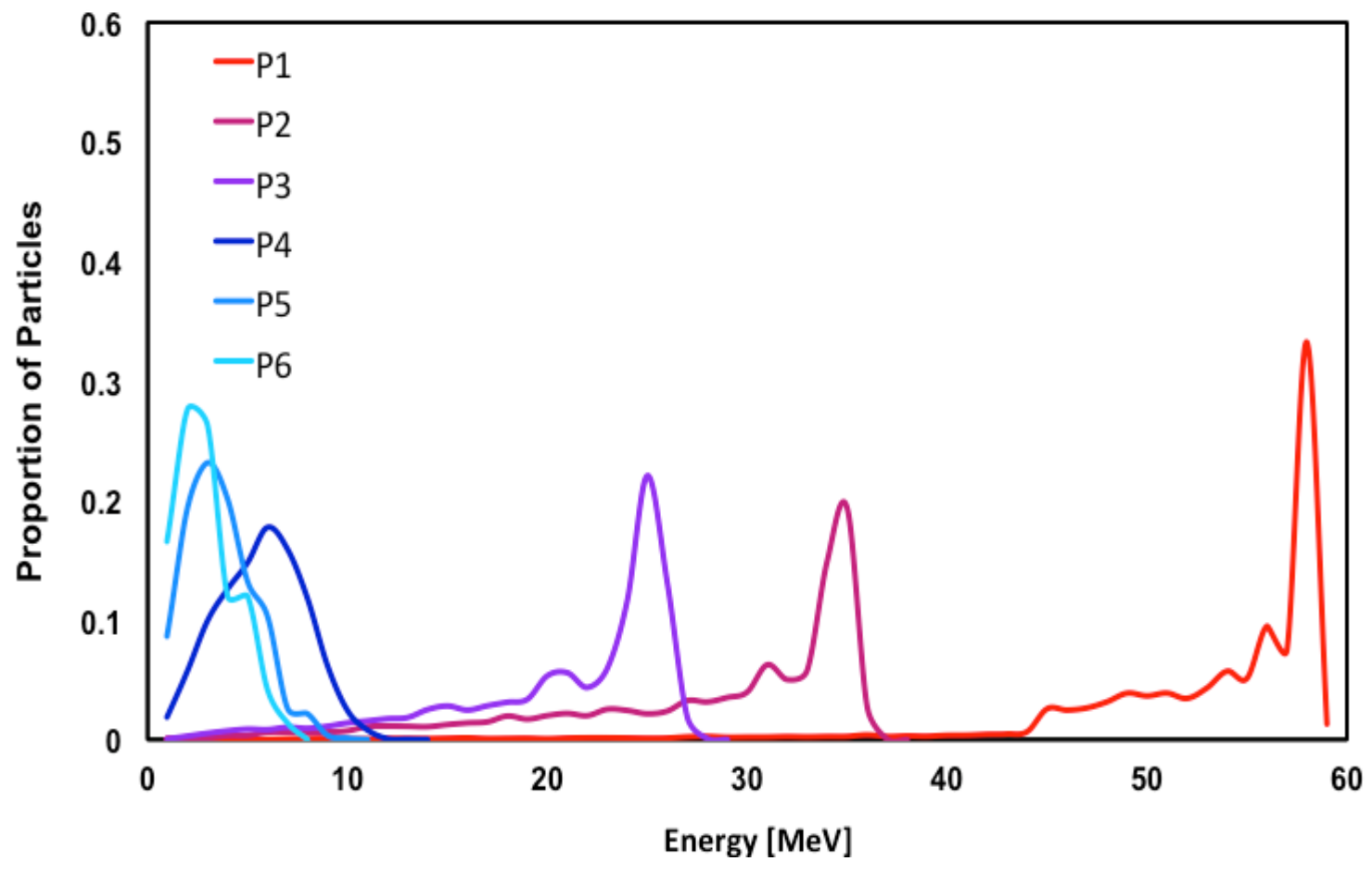


Supplementary Figure 2. Dependence of $\alpha$ and $\beta$ parameters on LET for normal fibroblast (AG01522, top panel) and radioresistant glioma (U87, bottom panel) cells exposed to a modulated $62 \mathrm{MeV}$ proton beam. $\alpha$ parameter increased linearly with increasing LET for both U87 and AG01522 cells. No statistically significant differences are observed for $\beta$ parameter for either monoenergetic or SOBP.
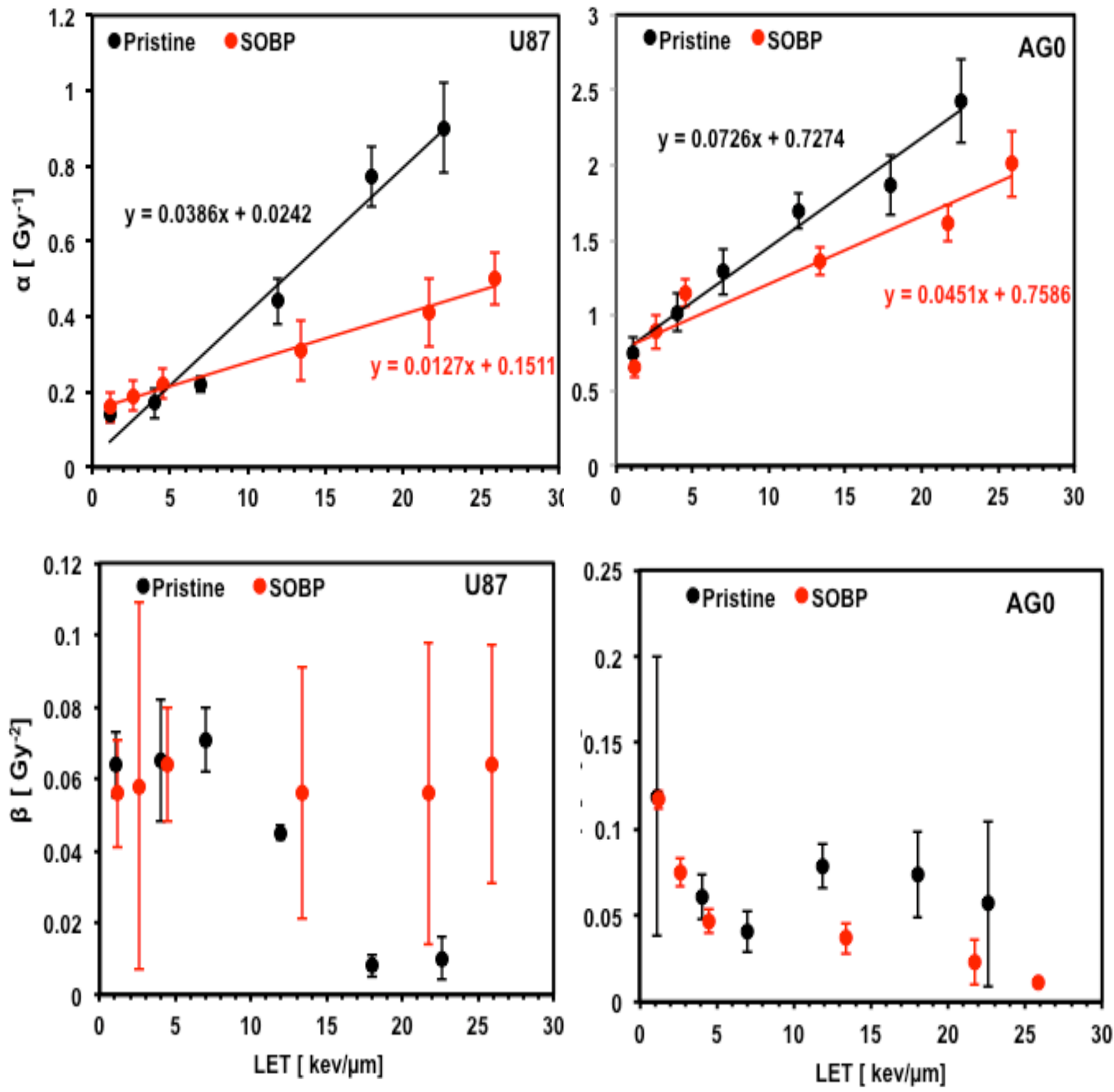